\section{(O) OPEN ACCESS}

\title{
Ischaemic stroke: the ocular motor system as a sensitive marker for motor and cognitive recovery
}

\author{
Wei Dong, ${ }^{1,2}$ Bernard Yan, ${ }^{2,3}$ Beth P Johnson, ${ }^{4}$ Lynette Millist, ${ }^{4}$ Stephen Davis, ${ }^{2,3,5}$ \\ Joanne Fielding, ${ }^{2,4,5}$ Owen B White ${ }^{2,3,4,5}$
}

\begin{abstract}
'Department of Neurology, West China Hospital, Sichuan University, Chengdu, Sichuan, China

${ }^{2}$ Department of Neurology, Royal Melbourne Hospital, Parkville, Victoria, Australia ${ }^{3}$ Department of Medicine, University of Melbourne, Parkville, Victoria, Australia ${ }^{4}$ Centre for Developmental Psychiatry and Psychology, School of Psychology and Psychiatry, Monash University, Clayton, Victoria, Australia ${ }^{5}$ Centre for Neuroscience, University of Melbourne, Parkville, Victoria, Australia
\end{abstract}

Correspondence to Associate Professor 0 B White, Department of Neurology, Royal Melbourne Hospital, Grattan St, Parkville, VIC 3050, Australia; owen.white@mh.org.au

Received 14 August 2012 Revised 30 October 2012 Accepted 31 October 2012 Published Online First 6 December 2012

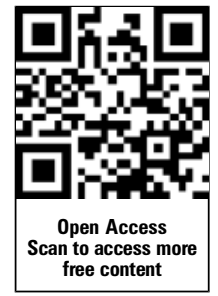

\section{SLinked}

- http://dx.doi.org/10.1136/ jnnp-2012-304481

- http://dx.doi.org/10.1136/ jnnp-2013-304886

\section{ABSTRACT}

Objective To evaluate the sensitivity of measuring cognitive processing in the ocular motor system as a marker for recovery of deficit in post stroke patients. Methods 15 patients (mean age 60.6 years, mean National Institutes of Health Stroke Scale (NIHSS) score 2.25) and 10 age matched control subjects (mean age 63.3 years) participated in the study. We included mildly affected acute stroke patients without a visual field defect or gaze palsy. Patients were examined at onset and at 1 month and 3 months post stroke by testing ocular motor function, NIHSS, modified Rankin Scale (mRS) and standard cognitive function assessments. Results Significant differences were found in measures of ocular motor function between groups at stroke onset as well as between the first test and follow-up in patients. At 3 months, function had not returned to normal baseline. Ocular motor function was more sensitive in identifying cognitive dysfunction and improvement compared with NIHSS or mRS.

Conclusions Standard neurological assessments of stroke patients are weighted significantly towards motor and sensory function, underestimating cognitive deficits. Ocular motor assessment demonstrates cognitive effects of even mild stroke and may provide improved quantifiable measurements of cognitive recovery post stroke. We demonstrated abnormality in patients just after onset, extending beyond 3 months, when there was apparent full recovery of motor and sensory function, implying more widespread disruption of cognitive mechanisms, consistent with the subjective complaints received from patients. This may provide insight into cognitive rehabilitation strategies leading to improved functional outcomes.

\section{INTRODUCTION}

Stroke is a major cause of morbidity and mortality, contributing significantly to the global health economic burden. ${ }^{1}$ The standard neurological assessment of stroke patients, the National Institutes of Health Stroke Scale (NIHSS), is weighted significantly towards motor and sensory function, ${ }^{2}$ consistently underestimating cognitive deficit that may be responsible for the subtle organisational dysfunction affecting many stroke patients. ${ }^{3}$

The ocular motor system is the first motor system to become functional in humans, and its organisation may be representative of sensorimotor integration in the somatic motor system. Whether or not this is true, anatomical substrate for motor function, and its higher order cognitive processes, occupy a significant amount of brain substance. ${ }^{4-9}$
Processes which disrupt brain tissue may be reflected in the assessment of cognitive processing required for ocular motor function. To this end, ocular motor studies, particularly those including paradigms to evaluate the cognitive processing of sensory input that influences motor output, have demonstrated significant characteristic abnormalities in degenerative diseases (Parkinson's disease $^{10-14}$ Huntington's disease ${ }^{14-16}$ ), developmental disorders (autism spectrum disorders ${ }^{17}$ ) and lesional diseases, such as multiple sclerosis. ${ }^{18-21}$

Cognitive processes, such as attention (prioritisation of sensory input), inhibitory control (the capacity to delay responses and ignore distraction), working memory, and learning and decision making processes, influence saccadic parameters. ${ }^{4}$ Moreover, although there is evident close correlation between studies of cognitive processing in ocular motor function and established cognitive tests, studies of ocular motor function have the advantage of evaluating the output effects of such deficits, and of quantifying them. Based on these observations it would seem that measures of the ocular motor system and its interactive cognitive systems may represent brain function more generally, potentially providing insight into the pathophysiology of dysfunctional states.

In the ocular motor system we are able to precisely control sensory input while being able to accurately measure motor output with modern eye tracking equipment. Major advantages include the limited degrees of freedom of movement of the eyes and the relative lack of complicating inertial influences. This provides a powerful tool for the investigation of neurocognitive and psychopathological processes in humans. ${ }^{6}$

Traumatic brain injury is, like stroke, usually a singular event. There are few studies of ocular motility in moderate or severe trauma but, in mild diffuse brain injury, ${ }^{22}$ abnormalities have been identified, characterised as impairment of response inhibition, visuospatial memory and attention. We suggest that ocular motor measures have the potential to be valuable in the assessment of neurological recovery post stroke. By demonstrating comparable sensitivity with the neurophysiological abnormalities seen in studies of other disease processes, they may provide quantifiable sensitive markers of both motor and cognitive deficits in stroke patients, especially relevant in those patients with good motor and language recovery but complaints of difficulty coping with a return to normal activity, possibly due to subtle cognitive deficits. 


\section{SUBJECTS AND METHODS Subjects}

Fifteen patients with a first ischaemic cerebral stroke, without a visual defect or gaze palsy, were recruited. Patients with mild stroke were preferentially selected as we wished to address the sensitivity of the techniques. Patients who met the criteria for selection were recruited consecutively. All were English speaking, not dysphasic or demented, and could understand and cooperate with the requirements of the assessment. Details of the patients are recorded in table 1. Mean NIHSS score was 2.25 (range 0-6). One of the patients was treated with intravenous tissue plasminogen activator at the time of recruitment and the NIHSS score was 0 at the time of evaluation for the study. Thirteen age matched neurologically healthy subjects, mean age 63.3 years (range 42-85), were recruited as a control group. They were derived from relatives of patients and hospital staff and volunteers. Ethics approval was granted by the Melbourne Health Human Research Ethics Committee, and all participants gave their informed consent prior to inclusion in the study, in accordance with the Helsinki declaration. All stroke patients continued with their normal treatment, five received specialised rehabilitation (table 1 ).

We did not attempt to differentiate patients with regards to anatomical location of the stroke and differential deficits that might have been observed with specific regions. This was considered beyond the scope of this study.

\section{Methods}

Given the exploratory nature of this study, we chose a comprehensive range of ocular motor assessments to capture performance over both volitional and more reflexive eye movements, incorporating a range of cognitive functions.

1. Visually guided saccades: assessment of the generation and control of more reflexive saccades, including an evaluation of attention, response suppression, predictive or anticipatory behaviours, and reprogramming of movement parameters. Participants generated $5^{\circ}$ or $10^{\circ}$ saccades to visual targets as they were illuminated, using unpredictable/predictable target sequences to modulate attentional state prior to saccade generation. The key measurements were saccade latency (ms), amplitude (degrees) and position error of the final movement.

2. Volitional saccades: assessment of the volitional generation of saccades (top down), including an evaluation of planning, response suppression and spatial working memory.

Table 1 Patient demographics

\begin{tabular}{ll}
\hline Feature & Patients $(\mathbf{n = 1 5})^{*}$ \\
\hline Age (years) & $60.6(45-86)$ \\
Gender (M/F) & $7 / 8$ \\
Infarct location & \\
Frontal lobe & 4 \\
Parietal lobe & 4 \\
Basal ganglia & 2 \\
Temporal lobe & 5 \\
Thalamus & 1 \\
Corona radiata & 1 \\
Rehabilitation & \\
Active rehabilitation & 4 \\
Without rehabilitation & 11 \\
\hline
\end{tabular}

*Note that two patients had more than one infarct site.
Participants generated $5^{\circ}$ or $10^{\circ}$ saccades to visual, imagined or remembered targets according to the experimenter's requirements, using:

A. antisaccades, whereby saccades were directed towards a spatial location in the opposite visual field to that of the stimulus; and

B. a memory guided protocol, whereby saccades were made to previously illuminated stimuli (working memory).

3. Standard clinical assessment using NIHSS score and the modified Rankin Scale (mRS) score at each testing time point.

4. Brief assessment of cognitive function using the Depression Anxiety Stress Scales (DASS), Backward Digit Span and Mini-Mental State Examination (MMSE).

For stroke patients, testing was conducted over three sessions, each lasting approximately $1 \mathrm{~h}$ :

1. Within 10 days of stroke

2. At 1 month post stroke

3. At 3 months post stroke.

Thirteen healthy controls were tested only once. It was felt that, although practise effects may occur, these would not be relevant in this setting where we were establishing deviation from normal function in patients post stroke.

\section{Statistical analysis}

To compare parameters between patients and controls at the time of the initial assessment, $t$ tests were used. Changes within stroke patients over the three testing sessions were assessed using a repeated measures analysis of variances (ANOVA) and non-parametric tests (eg, $\chi^{2}$ test for the error rate). The relationship between NIHSS, mRS and ocular motor measures was assessed using Pearson's 'r' correlations.

\section{RESULTS}

We will report here only those paradigms that showed significant deficits at onset, and demonstrated change in function over 3 months. In particular, these were measures of working memory, and planning and inhibitory control (memory guided saccades and antisaccades).

\section{Clinical and cognitive measurements}

Unfortunately, only nine control subjects underwent a full set of cognitive function tests compatible with those performed for the patient group. We did have eye movement data in a larger number, as indicated. We reviewed the statistics on eye movements using only subjects who completed all neuropsychological studies and there was no significant difference. Compared with the control group, stroke patients scored worse on tests of cognitive function. MMSE scores tended to be lower than absolute normal for age in patients but the reduction did not represent significant cognitive failure. It remains possible that more extensive testing in these domains might have shown more definable deficits across different domains. In this setting however, it was felt appropriate to proceed with less labour intensive clinical screening tools.

Patients consistently performed poorly on the Backward Digit Span. Similarly, there were elevated scores for depression, anxiety and stress in the patient group. Notably, there were no significant changes over the three time sessions for any of the cognitive measurements (table 2). We did note, however, that in contrast, the improvement in motor function was progressive, patient NIHSS scores decreasing significantly during the 1 month and 3 month follow-up periods ( $p=0.03$ and 0.01 , 
Table 2 Clinical and cognitive measurements in patients and control participants

\begin{tabular}{|c|c|c|}
\hline & $\begin{array}{l}\text { Patients }(n=15) \\
\text { (mean age } 60.6 \\
\text { (SD 15.5) years) }\end{array}$ & $\begin{array}{l}\text { Control }(\mathrm{n}=13) \\
\text { (mean age } 63.3 \\
\text { (SD 11.6) years) }\end{array}$ \\
\hline \multicolumn{3}{|l|}{ NIHSS } \\
\hline Onset (range) & $2.4(0-6)$ & $\mathrm{n} / \mathrm{a}$ \\
\hline 1 month (range) & $1.2(0-3) p=0.03^{*}$ & \\
\hline 3 months (range) & $1.2(0-2) p=0.01^{*}$ & \\
\hline \multicolumn{3}{|l|}{ MMSE } \\
\hline Onset & 27.8 & $29.6(n=10)$ \\
\hline 1 month & 28.1 & \\
\hline 3 months & 28.3 & \\
\hline \multicolumn{3}{|l|}{ Digit span } \\
\hline Onset & 15.5 & $18.6(n=9)$ \\
\hline 1 month & 17.7 & \\
\hline 3 months & 16.8 & \\
\hline \multicolumn{3}{|l|}{ DASS } \\
\hline \multicolumn{3}{|l|}{ Depression } \\
\hline Onset & 9.1 & $0.9(n=9)$ \\
\hline 1 month & 5.7 & \\
\hline 3 months & 9 & \\
\hline \multicolumn{3}{|l|}{ Anxiety } \\
\hline Onset & 6.9 & $0.4(n=9)$ \\
\hline 1 month & 4 & \\
\hline 3 months & 7 & \\
\hline \multicolumn{3}{|l|}{ Stress } \\
\hline Onset & 112 & $0.9(n=9)$ \\
\hline 1 month & 8.4 & \\
\hline 3 months & 5.7 & \\
\hline
\end{tabular}

respectively) (table 2). This raises the possibility of pre-existing cognitive pathology in patients prone to major cerebrovascular events, possibly on the basis of more widespread, pre-existing, previously asymptomatic, small vessel disease. That is beyond the scope of this current study to explore.

\section{Visually guided saccades}

These were well performed with normal latencies and saccade parameters (table 3 ). Mean amplitudes and mean absolute position error for stroke patients were normal relative to control participants.

Interestingly, the patient group did not show any degradation in performance in the presence of distractor stimuli (table 3). At time point 1, there was no difference between controls and stroke patients in latency, amplitude or mean absolute position error. This may be a reflection of the fact that we specifically selected patients with mild disease, but this could also be a statistical issue based on the diversity of anatomical pathology in the patient group.

There was no change over time in visually guided saccade performance in stroke patients on latency, amplitude or mean absolute position error (table 4).

\section{Antisaccades}

At the time of the initial assessment, stroke patients generated significantly more errors (inappropriate prosaccades to stimulus) than healthy controls $(\mathrm{t}(19)=3.68, \mathrm{p}=0.002)$
Table 3 Comparison of saccade variables between control and stroke patients at time point 1

\begin{tabular}{|c|c|c|c|c|}
\hline & \multicolumn{2}{|c|}{ Control } & \multicolumn{2}{|l|}{ Stroke } \\
\hline & Mean & SD & Mean & SD \\
\hline \multicolumn{5}{|l|}{ Visually guided task } \\
\hline Latency (s) & 0.19 & 0.03 & 0.22 & 0.09 \\
\hline Mean absolute position error & 9.8 & 3.62 & 8.93 & 5.14 \\
\hline \multicolumn{5}{|l|}{ Visually guided task with distractor } \\
\hline Latency (s) & 0.19 & 0.03 & 0.22 & 0.07 \\
\hline Mean absolute position error & 13.78 & 5.44 & 12.62 & 9.1 \\
\hline \multicolumn{5}{|l|}{ Antisaccade task } \\
\hline Latency (s) & 0.34 & 0.11 & $0.52^{*}$ & 0.22 \\
\hline Mean absolute position error & 42.58 & 24.5 & 33.9 & 24.6 \\
\hline \multicolumn{5}{|l|}{ Memory guided task } \\
\hline Latency (s) & 0.36 & 0.07 & 0.49 & 0.26 \\
\hline Mean absolute position error & 16.02 & 6.31 & 11.45 & 9.02 \\
\hline
\end{tabular}

(figure 1, table 3 ), and reaction times, as measured by saccade latency, were slower $(\mathrm{t}(19)=2.28, \mathrm{p}=0.034)$ (table 3 ).

There was no difference between patients and controls with respect to mean absolute position error. No correlation was found between error rate and other clinical measurements, such as NIHSS, DASS and MMSE, at the initial assessment in the stroke or control group. The error rate for stroke patients significantly improved in the first month $(p=0.019)$ relative to the initial assessment (figure 1). At 3 months their error rate had improved to a level that was not statistically significantly different from control participants (figure 1). This may be a manifestation of increased SD given that the mean error rate was greater in the patient group.

In summary, latency was prolonged initially, and error rate remained increased with patients being significantly more variable as a group than control subjects but showing a trend towards normal function.

Table 4 Changes in saccade variables in stroke patients over time

\begin{tabular}{|c|c|c|c|c|c|c|}
\hline & \multicolumn{2}{|l|}{ T1 } & \multicolumn{2}{|l|}{ T2 } & \multicolumn{2}{|l|}{ T3 } \\
\hline & Mean & SD & Mean & SD & Mean & SD \\
\hline \multicolumn{7}{|l|}{ Visually guided task } \\
\hline Latency (s) & 0.22 & 0.09 & 0.23 & 0.03 & 0.23 & 0.02 \\
\hline $\begin{array}{l}\text { Mean absolute position } \\
\text { error }\end{array}$ & 8.93 & 5.14 & 9.71 & 4.06 & 10.63 & 4.06 \\
\hline \multicolumn{7}{|c|}{ Visually guided task with distractor } \\
\hline Latency (s) & 0.22 & 0.07 & 0.23 & 0.07 & 0.23 & 0.07 \\
\hline $\begin{array}{l}\text { Mean absolute position } \\
\text { error }\end{array}$ & 12.62 & 9.1 & 9.65 & 4.89 & 12.81 & 6.31 \\
\hline \multicolumn{7}{|l|}{ Antisaccade task } \\
\hline Latency (s) & 0.52 & 0.22 & 0.50 & 0.24 & 0.46 & 0.30 \\
\hline $\begin{array}{l}\text { Mean absolute position } \\
\text { error }\end{array}$ & 33.90 & 24.60 & 27.92 & 15.14 & 27.72 & 15.75 \\
\hline \multicolumn{7}{|l|}{ Memory guided task } \\
\hline Latency (s) & 0.49 & 0.26 & 0.53 & 0.36 & 0.39 & 0.18 \\
\hline $\begin{array}{l}\text { Mean absolute position } \\
\text { error* }^{*}\end{array}$ & 11.45 & 9.02 & 9.58 & 4.52 & 10.71 & 5.23 \\
\hline
\end{tabular}




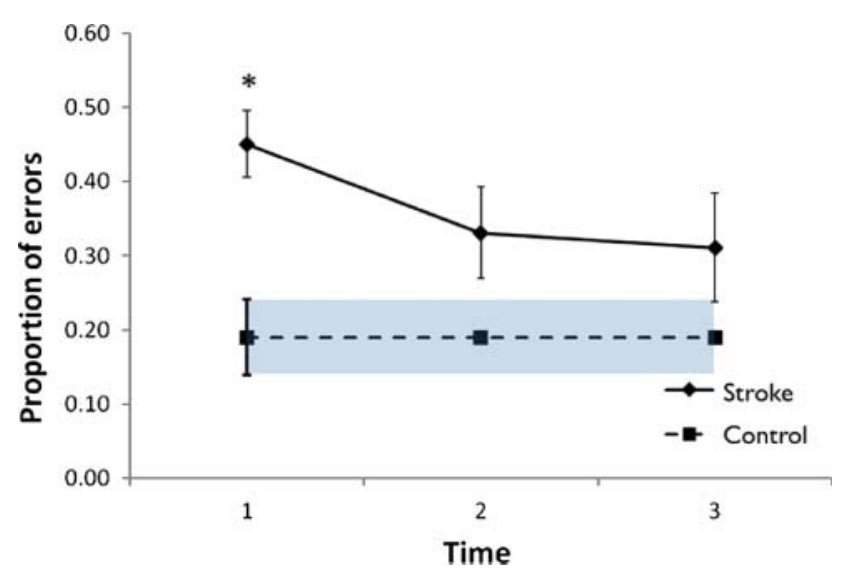

Figure 1 Proportion of errors in the antisaccade task in stroke patients. Data are represented as mean (SEM) for each time point. ${ }^{*} \mathrm{p}<0.005$.

\section{Memory guided saccades}

Patients made significantly more errors than healthy controls at the time of the initial assessment $(p=0.001)$. Repeated measures ANOVA indicated that there was a significant improvement in error rate from the time of initial assessment to the final assessment $(p=0.035)$ (figure 2$)$. The patient mean error rate remained higher than the control group but was not statistically different from normal. There was no difference between groups in mean absolute position error (table 3). Similar to the antisaccade study, no correlation was found between error rate and other clinical measurements at the time of the initial assessment.

\section{DISCUSSION}

There are currently no studies of ocular motor tests being used to document cognitive deficits in acute stroke patients and their neurological recovery post stroke. However, patients, even with mild strokes, often complain of 'not being normal' for quite some months after stroke. Cognitive deficits have been identified in stroke patients. ${ }^{23-25}$ Although this was not primarily a study of conventional tests of cognitive function, in this limited study, patients had higher scores for depression than would be considered normal. There was no longitudinal improvement in these scores over 3 months. This is consistent with reports showing that depression is more commonly present in stroke patients and not infrequently worsens over

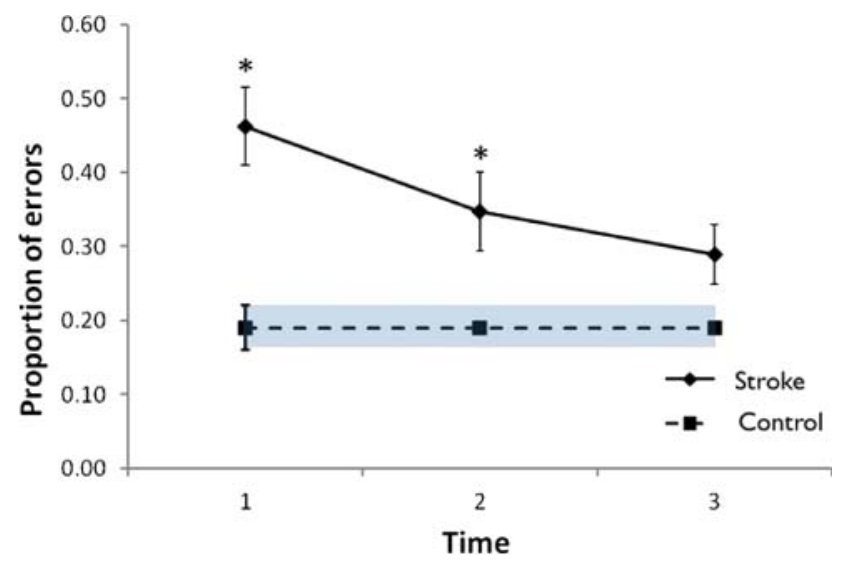

Figure 2 Proportion of errors in the memory guided task in stroke patients. Data are represented as mean (SEM) for each time point. ${ }^{*} p<0.005$. time following stroke. ${ }^{26}$ The presence and persistence of depression and abnormal scores for cognitive function may reflect preexisting pathology, possibly vascular, in this group of patients, and may not simply result from the stroke. ${ }^{25}$

The question arises as to whether depression alone could explain the abnormalities observed. There is a paucity of literature on this but, in previous studies undertaken in this laboratory in melancholic and non-melancholic depression, ${ }^{27} 28$ the primary abnormalities were slightly elevated peak velocity amplitude in non-melancholic depressives for larger saccades and lowered peak velocities in melancholic patients. No abnormalities of memory guided saccades were seen in either group. Melancholic depressives showed some inaccuracy of recalculated saccades after unpredictable 'odd ball' changes in target amplitude and direction. Antisaccades were not specifically investigated. The pattern of results was not reminiscent of those seen in our patients and, although not entirely excluding the possibility of a depression contributing, these results would support our contention that our findings represent true changes in cognitive processing of ocular motor responses secondary to stroke related pathology.

Although the stroke patients enrolled in this study had relatively low NIHSS and mRS scores, their ocular motor test parameters showed quantifiable abnormalities, especially early in the course of the recovery period, with progressive change, towards normal, especially in the two most robust areas of abnormality, the performance of memory guided saccades and antisaccades. Significantly, performance of these tasks is dependent on extensive activity in complex and diffuse circuits, which we might expect to be more susceptible to even minor disruption.

The absence of inhibitory errors to distractors may be a manifestation of task complexity. The shorter mean latencies over time, in memory guided protocols, may indicate either a subsequent reduction in inhibitory control or possibly facilitation of release of a target of regard indicating impairment of more complex circuitry involved in attentional shifts and eye movement control.

We did not demonstrate an abnormality in visually guided saccadic function. This may be a manifestation of the fact that pathology was diverse and that inhibitory errors may be more evident with further studies of more specific pathology or with larger numbers of subjects.

Of note is that these findings did not correlate with other clinical measurements, indicating that that these quantifiable tests of input, cognitive processing and output may be independent or, more likely, more subtle markers of brain dysfunction than NIHSS and mRS. This should be interpreted with some caution, however, as the spread of scores for cognitive testing was narrow and there is a known ceiling effect with MMSE scores in particular.

Similar studies in multiple sclerosis have shown sensitivity of ocular motor paradigms and are showing potential for documenting disease progression. ${ }^{18-21}$ It may be that ocular motor testing, which is simple and cheap, may ultimately be a more effective way of assessing widespread neurological disease given the difficulties of accessing comprehensive neuropsychological assessment in a timely manner.

These studies raise the possibility of conducting wider ranging studies. Ocular motor studies addressing cognitive function may also provide insight into the vague feelings of dysfunction complained of by many patients following cardiac bypass surgery. They may help characterise early deficits in patients with substantial subcortical ischaemic disease, identified incidentally but 
still functioning in the community. It remains uncertain whether such findings are representative of static or dynamic disease processes. Studies such as these may provide evidence not only of deficit but possibly progression, which would indicate a more aggressive approach to therapy.

This study has demonstrated the capacity to document change in these mildly affected patients using a small sample of potential studies. We would note that we have not undertaken the extensive cognitive testing required to validate cognitive abnormality in our patient group, all of whom were mildly affected neurologically. Such a deficit has been demonstrated ${ }^{23-25}$ but requires extensive testing over a $2 \mathrm{~h}$ period, with subsequent analysis, and is highly resource dependent. By comparison, our ocular motor data are collected and analysed in less than an hour of testing, undertaken by a technician. Our results suggest that there is subtle cognitive dysfunction, reflected in the ocular motor system, that persists for months, even after mild stroke, seemingly independent of the apparent improvement in motor and primary sensory function. Rather than this being evidence of independence of the two systems, ocular motor and somatic motor, it more likely represents a lack of sensitivity of functional testing of somatic motor parameters in a clinically convenient manner.

It may well be that cognitive retraining of attentional systems, especially in the early stages of recovery, may help recovery overall and facilitate the return towards more normal function as well as patient perception of well being.

Further large studies are needed to confirm these findings and explore the correlation between ocular motor studies and the pathophysiology of different types of stroke. The finding of specific abnormalities on tests of cognitive function, persistent throughout the experimental period, does raise the possibility of more longstanding mild cognitive impairment in at least some of these patients. Given that the risk factors for small vessel disease and stroke are essentially the same, it remains possible that there may be pre-existing small vessel disease, predisposing to both stroke and cognitive impairment, at a much earlier stage than we have hitherto suspected. Our patients did not have radiological evidence of extensive small vessel disease but it might be that further studies of patients with relatively mild disease on radiological studies merit further investigation and characterisation of deficits. Such tests as we have performed in these studies may be a sensitive, accurate and inexpensive means of testing otherwise asymptomatic patients who come to attention for incidental reasons. It is possible that early aggressive intervention may prevent deterioration. This is an area that deserves further exploration.

Contributors WD: recruitment, experimental data collection, analysis of the experimental data and initial draft of the paper as well as review of the final version. BY: initial design of the paper, acquisition and analysis of the clinical data, and initial drafting of the paper as well as review of the final version. BPJ: statistical analysis of the experimental data, construction of the tables and figures, and final drafting of the paper. LM: data collection and supervision of WD during data collection and analysis. She has also reviewed all drafts of the paper. SD: acquisition of the clinical data and analysis of the stroke data as well as writing of the paper, including the final draft submitted. JF: initial design of the study, analysis of the eye movement data and writing all versions of the paper, including the final draft. OBW: design of the original study, data analysis and wrote several versions of the paper, including the current version, with the assistance and advice of all of the coauthors.

Funding Partial support for patient transport was provided by the Royal Melbourne Hospital Research Fund.

Competing interests None

Ethics approval Ethics approval was provided by the Melbourne Health Human Research Ethics Committee.

Provenance and peer review Not commissioned, externally peer reviewed.
Open Access This is an Open Access article distributed in accordance with the Creative Commons Attribution Non Commercial (CC BY-NC 3.0) license, which permits others to distribute, remix, adapt, build upon this work non-commercially, and license their derivative works on different terms, provided the original work is properly cited and the use is non-commercial. See: http://creativecommons.org/ licenses/by-nc/3.0/

\section{REFERENCES}

1. Murray CJ, Lopez AD. Mortality by cause for eight regions of the world: Global Burden of Disease Study. Lancet 1997;349:1269-76.

2. Goldstein LB, Bertels C, Davis JN. Interrater reliability of the NIH stroke scale. Arch Neurol 1989;46:660-2.

3. Woo D, Broderick JP, Kothari RU, et al. Does the National Institutes of Health Stroke Scale favor left hemisphere strokes? NINDS t-PA Stroke Study Group. Stroke 1999:30:2355-9

4. McDowell JE, Dyckman KA, Austin BP, et al. Neurophysiology and neuroanatomy of reflexive and volitional saccades: evidence from studies of humans. Brain Cogn 2008;68:255-70.

5. Pierrot-Deseilligny C, Muri RM, Ploner CJ, et al. Cortical control of ocular saccades in humans: a model for motricity. Prog Brain Res 2003;142:3-17.

6. Hutton SB. Cognitive control of saccadic eye movements. Brain Cogn 2008;68:327-40.

7. Hutton SB, Ettinger U. The antisaccade task as a research tool in psychopathology: a critical review. Psychophysiology 2006;43:302-13.

8. Carpenter RH. Frontal cortex. Choosing where to look. Curr Biol 1994;4:341-3.

9. Milea D, Lobel E, Lehericy $S$, et al. Cortical mechanisms of saccade generation from execution to decision. Ann N Y Acad Sci 2005;1039:232-8.

10. Chan F, Armstrong IT, Pari G, et al. Deficits in saccadic eye-movement control in Parkinson's disease. Neuropsychologia 2005;43:784-96.

11. Cameron IG, Watanabe M, Pari G, et al. Executive impairment in Parkinson's disease: response automaticity and task switching. Neuropsychologia 2010;48:1948-57.

12. Winograd-Gurvich C, Georgiou-Karistianis N, Fitzgerald PB, et al. Self-paced saccades and saccades to oddball targets in Parkinson's disease. Brain Res 2006:1106:134-41.

13. Fielding J, Georgiou-Karistianis N, Bradshaw J, et al. No sequence dependent modulation of the Simon effect in Parkinson's disease. Brain Res Cogn Brain Res 2005;25:251-60.

14. Fielding J, Georgiou-Karistianis N, Millist L, et al. Temporal variation in the control of goal-directed visuospatial attention in basal ganglia disorders. Neurosci Res 2006:54:57-65.

15. Leigh RJ, Folstein SE, Lasker AG, et al. Abnormal ocular motor control in Huntington's disease. Neurology 1983;33:1268-75.

16. Blekher T, Johnson SA, Marshall BS, et al. Saccades in presymptomatic and early stages of Huntington disease. Neurology 2006;67:394-9

17. Stanley-Cary C, Rinehart N, Tonge B, et al. Greater disruption to control of voluntary saccades in autistic disorder than Asperger's disorder: evidence for greater cerebellar involvement in autism? Cerebellum 2011;10:70-80.

18. Fielding J, Kilpatrick T, Millist L, et al. Longitudinal assessment of antisaccades in patients with multiple sclerosis. PLoS One 2012;7:e30475.

19. Fielding J, Kilpatrick T, Millist L, et al. Control of visually guided saccades in multiple sclerosis: disruption to higher-order processes. Neuropsychologia 2009;47:1647-53.

20. Fielding J, Kilpatrick T, Millist L, et al. Antisaccade performance in patients with multiple sclerosis. Cortex 2009;45:900-3.

21. Fielding J, Kilpatrick T, Millist L, et al. Multiple sclerosis: cognition and saccadic eye movements. J Neurol Sci 2009;277:32-6.

22. Heitger $\mathbf{M H}$, Jones RD, Macleod AD, et al. Impaired eye movements in post-concussion syndrome indicate suboptimal brain function beyond the influence of depression, malingering or intellectual ability. Brain 2009;132:2850-70.

23. Popovic IM, Seric V. Demarin V. Mild cognitive impairment in symptomatic and asymptomatic cerebrovascular disease. J Neurol Sci 2007;257:185-93.

24. Rao R, Jackson S, Howard R. Neuropsychological impairment in stroke, carotid stenosis, and peripheral vascular disease, A comparison with healthy community residents. Stroke 1999:30:2167-73.

25. Mok VC, Wong A, Lam WW, et al. Cognitive impairment and functional outcome after stroke associated with small vessel disease. J Neurol Neurosurg Psychiatry 2004; 75:560-6.

26. Brodaty H, Withall A, Altendorf A, et al. Rates of depression at 3 and 15 months poststroke and their relationship with cognitive decline: the Sydney Stroke Study. Am J Geriatr Psychiatry 2007;15:477-86.

27. Winograd-Gurvich C, Georgiou-Karistianis N, Fitzgerald PB, et al. Self-paced and reprogrammed saccades: differences between melancholic and non-melancholic depression. Neurosci Res 2006;56:253-60.

28. Winograd-Gurvich C, Georgiou-Karistianis N, Fitzgerald PB, et al. Ocular motor differences between melancholic and non-melancholic depression. J Affect Disord 2006:93:193-203. 